\title{
Die aktuelle Bedeutung des Subjektaspekts für Ideologieproduktion und Ideologiekritik: Menschliche Subjektivität als Teilaspekt der "gesellschaftlichen Natur" des Menschen
}

\section{Renatus Schenkel}

Keywords: Ideologierezeption, Kritische Psychologie, individuelle Medialkommunikation, Reduktionismus, individuelle Subjektivität, marxistische Subjekttheorie, marxistische Medientheorie

\section{Abstract}

Für das qualitative Verständnis der Wechselwirkung von Ideologieproduktion und Ideologienutzung ist die Kenntnis der konkreten Verwendungsmodi von ideologischen Angeboten durch Individuen unverzichtbar. Als diskursive, lautliche oder ikonische Bedeutungskonstellationen sind Ideologieangebote nicht mehr nur determinierende Handlungsbedingungen sondern werden zu Handlungsmöglichkeiten, die in entwickelten kapitalistischen Gesellschaften auf widersprüchliche Weise individuell genutzt, abgelehnt oder nicht zur Kenntnis genommen werden. Auf Basis der Kritischen Psychologie als am weitesten entwickelter marxistischer Subjekttheorie und von marxistischer Medientheorie liefert der nachfolgende Beitrag subjekttheoretische Bausteine zur kritischen Aufklärung grundlegender individueller Wirkungsmechanismen von Ideologieproduktion.

Schenkel, Renatus. 2019. „Die aktuelle Bedeutung des Subjektaspekts für Ideologieproduktion und Ideologiekritik: Menschliche Subjektivität als Teilaspekt der ,gesellschaftlichen Natur' des Menschen“. In Ideologie, Kritik, Öffentlichkeit. Verhandlungen des Netzwerks Kritische Kommunikationswissenschaft, herausgegeben von Uwe Krüger und Sebastian Sevignani, 320-346. Universität Leipzig. DOI: 10.36730/ideologiekritik.2019.15

Renatus Schenkel |HS Magdeburg-Stendal | renatus.schenkel@hs-magdeburg.de 


\section{Einführung}

„Zu verstehen, wie Denk- und Sprachformen für die Herstellung, Aufrechterhaltung und Rechtfertigung von Macht- und Herrschaftsverhältnissen funktional sind“", so ein Ziel der Tagung, aus der dieser Beitrag hervorgegangen ist, erfordert - neben dem Wissen über die gesellschaftlichen Grundlagen der Produktion und der Herrschaftsverwendung von Ideologie - auch die Kenntnis ihrer individuellen Verwendungszusammenhänge. Dieser letztere Subjektaspekt ist aus verschiedenen historischen, aber auch theoretischen Gründen in der kritischen wie marxistischen Diskussion nach wie vor unterbelichtet, in Teilen der traditionellen Medienforschung wird er problematisch verkürzt. Anhand vorhandener Erklärungsdefizite möchte ich vor dem Hintergrund des aktuellen Standes der Kritischen Psychologie - als am weitesten entwickelter marxistischer Subjektwissenschaft - deren Erkenntnispotenziale für eine marxistisch fundierte Ideologiekritik ausleuchten ${ }^{1}$.

Da die Kritische Psychologie als eigenständiger Ansatz in der Vielzahl kritischer Psychologieansätze hier vermutlich nur Wenigen bekannt ist und sie - vor allem auf Grund politischer Invektiven ${ }^{2}$ in den 1970er Jahren - bislang nur bruchstückhaft für Medien- und Kommunikations-

1 Durch die Überarbeitung des Vortragsmanuskripts ergab sich die Gelegenheit, dieses noch um einige Aspekte zu ergänzen, was gleichwohl in dem verbliebenen Zeitraum nichts an dem nach wie vor skizzenhaften Charakter mancher Sequenzen ändern konnte. In diesem Zusammenhang möchte ich Eric Recke danken, der mir mit inhaltlichen Anregungen und formaler Hilfe die Bearbeitung deutlich erleichtert hat.

2 Gemeint sind Berufsverbote für marxistische Wissenschaftler, Nichtberufungen, wodurch vielversprechende Ansätze vor einigen Jahrzehnten abgebrochen wurden und sich so eine marxistische Tradition an deutschen Hochschulen, wie sie in anderen Ländern wie z. B. in Großbritannien üblich ist, nicht etablieren konnte. Die Kritische Psychologie als auch international beachteter Wissenschaftsansatz wurde explizit durch Streichung zum Beispiel der Holzkamp-Professur nach dessen Emeritierung trotz einer über die verschiedenen Lager hinweg einvernehmlich verabschiedeten Berufungsliste und Berufsverboten für Kritische Psychologen durch den Westberliner Wissenschaftssenator und Kommunikationswissenschaftler (!) Glotz und die SPD-Kader im Westberliner Senat stark behindert. Zwar sprechen politische Gegner marxistisch orientierter Organisationen immer gern von deren „Kaderpolitik“. Bei diesen Berufsverbotsverfahren (PR-Bezeichnung: „Radikalenerlass“) ist diese Formulierung ebenso angebracht. Denn natürlich waren auch partei- und wissenschaftspolitisches Konkurrenzdenken ein Motiv für solche undemokratischen wissenschaftspolitischen Eingriffe, die die proklamierte Wissenschaftsfreiheit ad absurdum führte. Vgl. Holzkamp (1977a), Maiers und Markard (1978) sowie Meyen (2017). 
wissenschaft genutzt werden konnte ${ }^{3}$, möchte ich zwecks besserer Einordnung in den interdisziplinären Gesamtkontext der Fragestellung zunächst einige wissenschaftspolitische und -theoretische Bemerkungen voranschicken, um dann wieder zur eigentlichen Fragestellung zurückzukehren. Um Verwechslungen mit anderen kritischen psychologischen Ansätzen zu vermeiden, schreibt sich der Ansatz der Kritischen Psychologie mit großem $\mathrm{K}$.

Immerhin hat sich die Kritische Psychologie, die traditionelle Psychologie aus inneren Widersprüchen heraus überschreitend (vgl. Maiers 1988a und 1988b), flankiert durch eine damals starke Studentenbewegung und massiver politischer Bekämpfung durch SPD-geführte Berliner Hochschulsenatoren trotzend (vgl. Anm. 2), durch Aufarbeitung der für das Begreifen menschlicher Psyche relevanten Forschungsergebnisse anderer Einzelwissenschaften wie der Biologie, durch theoretisch vermittelte Rückgriffe auf die Evolutionstheorie und auf die Erkenntnisse des historischen und dialektischen Materialismus und der marxistischen Kritik der Politischen Ökonomie zu einem positiven Gegenkonzept entwickeln können (Holzkamp 1983, Holzkamp-Osterkamp 1976, Maiers 1985/2, Schurig 1975, Maiers 1999).

Ermöglicht wurde dies vor allem durch die Entwicklung des epistemologisch und methodologisch grundlegend neuen Verfahrens der so genannten funktional-historischen Analyse des Mensch-Welt-Zusammenhangs. Erkenntnisleitend war "die Herausarbeitung der biologischen Voraussetzungen menschlicher Gesellschaftlichkeit durch Ableitung aus deren phylogenetischen Entstehungsbedingungen, also sozusagen die Erfassung der, gesellschaftlichen Natur' des Menschen in ihren verschiedenen funktionalen Aspekten." (Holzkamp 1977 b, 417-418). Erst damit sah sich die Kritische Psychologie zunehmend in in den Stand versetzt, die aus der Vernachlässigung der phylogenetischer Grundlagen psychischer Funktionsaspekte resultierenden grundsätzlichen Defizite der bürgerlichen Psychologie zu überwinden.

Das so fundierte Gegenkonzept knüpfte hierbei an die grundlegende Kritik der bürgerlichen Psychologie durch den zur kulturhistorischen Schule zählenden sowjetischen Psychologen A.N. Leontjew an. Deren

3 Explizit rekurrierieren auf die Kritische Psychologie in der Medien- und Kommunikationstheorie historisch wie aktuell Holzer 1974/1978, Hoffmann 1976, Schenkel 1984/2019 und Sevignani 2019, vgl. Anmerkung 14. 
Kernstück ist „die Zurückweisung [...] des so genannten ,Unmittelbarkeitspostulats' der bürgerlichen Psychologie, [...] ihre Auffassung von der (lediglichen, R.S.) ,Zweigliedrigkeit' der Beziehung des Menschen zur Welt als Hervorbringung von Reaktionen der Individuen auf Grund unmittelbarer Einwirkungen aus der Umwelt. [...] Dies ist die Basis für eine scharfe Kritik am Reduktionismus der bürgerlichen Psychologie, besonders als biologistische Einebnung der menschlichen, also gesellschaftlichen Spezifik der Lebenstätigkeit auf das organismische Niveau hin (aber ebenso auch als 'soziologistische' Reduktion), womit eine solche Psychologie die zentralen Probleme der gesellschaftlichen Existenz von Individuen von vornherein verfehlen muß" (Holzkamp 1982, 2-3, sowie allgemein Leontjew 1977). Auch in der Kommunikationswissenschaft lassen sich vergleichbare Probleme verorten, da zusammen mit anderen Anleihen auch dieser Reduktionismus aus der traditionellen Psychologie übernommen wurde, ohne deren innerwissenschaftliche Kontroversen zu reflektieren und gegebenenfalls entsprechende Konsequenzen für die Kommunikationswissenschaften zu ziehen. Die Folge ist ein verkürzter Begriff individueller Subjektivität, der eine adäquate Erfassung des individuellen Umgangs mit Medien und mit ideologisch geprägten Denkformen erschwert, wenn nicht verhindert. Ich komme darauf später zurück.

\section{Marxistische Medientheorie und Subjekttheorie der Kritischen Psychologie}

In den Kommunikations- und Medienwissenschaften kam es darüber hinaus auf Grund noch anderer innerwissenschaftlicher Gegebenheiten und ebenfalls massiver politischer Eingriffe - berüchtigtes Beispiel ist das verfassungswidrige Berufsverbot für den renommierten Kommunikationswissenschaftler Horst Holzer mit Verlust seiner Professur in München gar nicht erst zu einer weiter entwickelten marxistischen Medienkonzeption, obwohl Ansätze von Horst Holzer, Franz Dröge, Wulf D. Hundt, Burkhard Hoffmann, Volker Gransow, Jochen Bach, Chup Friemert und anderen ebenfalls vielversprechend waren. Gleichwohl hatte es noch eine Phase der Annäherung kritischer kommunikationstheoretischer Ansätze an die Kritische Psychologie z. B. durch Horst Holzer, Burkard Hoffmann, mir und einige andere gegeben. Wir hatten beispielsweise Holzer für Gastvorträge am Institut für Publizistik der FU Berlin gewinnen können 
(vgl. etwa Holzer 1978), mit Chancen auf eine Professur, die aber durch politische und interne Intrigen unter anderem des dort damals dominierenden, heute gern als liberal bezeichneten Professors zunichte gemacht wurden.

In der Spätphase seines Wirkens hat Horst Holzer in Aufarbeitung des damaligen Standes der Kritischen Psychologie die gleichrangige Bedeutung der gesellschafts- wie der persönlichkeitstheoretischen Kritik an traditioneller Kommunikations- und Medientheorie hervorgehoben. So formulierte er etwa auf dem 4 . Kongress für Kritische Psychologie in seinen Thesen zur Fruchtbarkeit des kritisch-psychologischen Ansatzes, dass auf dieser Basis zweierlei zu leisten sei:

- Kritik an den herrschenden positivistischen kommunikationswissenschaftlichen Positionen und

- die Entwicklung einer alternativen, historisch-materialistisch fundierten Kommunikations- und Medientheorie.

Kritik wie Alternative seien dabei sowohl gesellschaftstheoretisch wie persönlichkeitstheoretisch auszurichten (Holzer 1977, 449). Anzusetzen sei bei verhaltens- und handlungstheoretischen Konzepten von Kommunikation und Massenkommunikation und „dabei auf den Angelpunkt materialistischer Kommunikationstheorie zu beziehen: auf die dialektische Verschränktheit von Arbeits-, Erkenntnis- und Kommunikationsprozessen und deren Verankerung im Stoffwechselprozeß Mensch/Natur, damit auf diesen Stoffwechselprozeß als materiell begründetem, kommunikativ-kooperativem Handlungsvollzug" (ebd., 450).

Die Beschäftigung mit dem Verhalten des Individuums zu den gesellschaftlichen Bedeutungskonstellationen als Teil des Spezifikums menschlicher Subjektivität - die ideologischen Angebote in der bürgerlichen Gesellschaft sind als Denkformen und Symbolbedeutungen Teil dieser Konstellationen - kann also nicht nur nachgeordnet, quasi abgeleitet von gesellschaftstheoretischen Positionen oder gar in diesen schon als aufgehoben geltend geschehen, sondern muss ganz im Sinne von Holzers Erkenntnissen methodisch bereits persönlichkeitstheoretisch, und das bedeutet: subjekttheoretisch fundiert (siehe Anm. 3) erfolgen und sich ebenfalls der phylogenetischen Grundlagen kommunikativen und medialen Handelns - als naturhistorischer Prozess des Stoffwechsels - annehmen, um die kommunikativen Anteile bei Herausbildung und Stabilisierung 
der gesellschaftlichen Natur des Menschen bis hin in die kapitalistische Gegenwart herausarbeiten zu können. ${ }^{4}$

In Bezug auf ihren psychologischen Gegenstand hat die Kritische Psychologie dies wie bereits erwähnt unter Rückgriff auf grundlegende phylogenetische und anthropogene Entwicklungsansätze und - bezüglich gesellschaftlicher Evolution - auf Basis der marxistischen Gesellschaftstheorie unternommen, weshalb sie sich ihrem eigenen Anspruch nach auch als eine marxistisch fundierte subjektwissenschaftliche Psychologie (Maiers 2019, 131) versteht. Erst durch den Rückgriff auf die Evolutionstheorie und mit dem Blick auf die über drei Millionen Jahre währende Entwicklung von den ersten Australopithecinen bis hin zum Jetzt-Menschen (homo sapiens sapiens) wird nämlich sichtbar, dass dessen biologisch-genetische Natur ihn als einziges existierendes Lebewesen befähigt, aber auch zwingt, gesellschaftlich zu leben und dies in einem dialektischen Prozess von Vergegenständlichung und Aneignung der vorgefundenen Lebensbedingungen einschließlich aller Lebens-,Mittel' im umfassenden Sinn zu tun mit dem Ergebnis der Schaffung einer überdauernden externen Lebensumwelt.

Diese materiell-gegenständliche Lebensumwelt wird nach Durchsetzung des historisch-gesellschaftlichen gegenüber dem naturhistorischen Prozess zum überdauernden materiellen Träger der menschlichen Traditionsbildung. Die übergreifenden zentralen Triebkräfte der gesellschaftlich-historischen Entwicklung sind dann gemäß Marx und Engels nicht mehr primär durch natur-evolutionäre Mechanismen bestimmt, obwohl Evolution natürlich weiter wirkt, sondern durch eine neue Dialektik von Produktivkräften und Produktionsverhältnissen, deren jeweils historisch unterschiedlicher Stand die spezifische Entwicklungsdynamik einer historischen Formation ausmacht, also durch eine gesellschaftlich-

Holzkamps Analysen der Lern- und Aneignungsprozesse von Gegenstands- und Symbolbedeutungen durch Wahrnehmung zeigen zum Beispiel, wie unter bürgerlich-kapitalistischen Verhältnissen bereits in der frühen Kindheit die notwendige Aneignung der sich widersprechenden Gebrauchswert- und Tauschwertcharakteristika als Bedeutungen zu einem verkürzten Weltverständnis führt, das den Boden für bürgerliche Ideologieentstehung und -wirkung bildet, die erst durch eine Umstrukturierung von Denken und Wahrnehmung im begreifenden Denken überwunden werden kann (Holzkamp 1973, 233ff., ders. 1983, 317, 364ff. und 400f.). Zum Begriff des Ideologischen aus Sicht der Kritischen Psychologie in Abhebung zu anderen Ansätzen vgl. H.-Osterkamp (1983, 7ff.). 
evolutionäre Entwicklung, die gleichwohl nach wie vor als Teil der natürlichen Evolution - als diese in neuer Qualität - zu verstehen ist, da die Menschen ja Naturwesen - als einzige auf dem Planeten auf diesem neuen Niveau nach Aussterben beziehungsweise genetischer Absorption des Neander-thalers (homo sapiens neanderthalensis) - bleiben und dies in zunehmender Wechselwirkung mit den übrigen Lebewesen. Auch aus diesem Aspekt heraus ergibt sich jenseits unmittelbar ethischer Argumente und der mittlerweile unübersehbaren direkten Auswirkungen menschlicher Tätigkeit auf deren Lebensqualität eine tieferer Zusammenhang für die planetare Verantwortlichkeit des Menschen bei jeglicher Thematik, ob dies Klima, Artenvielfalt, Atmosphärenzusammensetzung und anderes mehr betrifft.

Der menschliche Lebensvollzug gestaltet sich innerhalb sozialer und darüber hinaus gehender kooperativer Beziehungen mit den anderen Menschen fürderhin als Teil des gesellschaftlichen Stoffwechselprozesses in Wechselwirkung mit der gegenständlich-materiellen Welt. Dabei sind vom Individuum von Geburt an und genetisch zwingend seine konkret es umgebenden gesellschaftlich-gegenständlichen Bedeutungs- und anderen Strukturen anzueignen und im fortschreitenden Sozialisationsprozess zunehmend aktiv mitzugestalten und umzuändern, nur so kann nach Auffassung der Kritischen Psychologie das Individuum seine persönliche Subjektivität entfalten. Ist dieser Prozess behindert, kommt es zu fundamentalen Defiziten, wie dies mit dem bekannten Beispiel von Kaspar Hauser illustriert werden kann. ${ }^{5}$ Die Summierung aller dieser Aktivitäten der lebenden Individuen bewirkt letztlich die historische Weiterentwicklung ihrer Produktionsverhältnisse und -mittel; es muss damit nicht mehr auf ein wie auch immer geartetes gesondertes Movens wie etwa den Hegelschen „Weltgeist" oder andere, übersinnlich-mystische Ursachen als Erklärungsfaktor für gesellschaftliche Entwicklung zurückgegriffen

$5 \quad$ Kaspar Hauser war angeblich als Kind im Wald ausgesetzt oder auch in einem Verlies eingesperrt worden und ohne jeglichen menschlichen sozialen Kontakt aufgewachsen, bis er im Alter von 16 Jahren auf eine menschliche Gemeinschaft traf. Dadurch litt er unter fundamentalen personellen Defiziten. Sie werden heute in der Psychologie als besondere beziehungsweise extremste Form des so genannten Hospitalismus gefasst, mit dem die negativen Folgen eines länger anhaltenden Entzugs körperlicher und psychischer Reize, sei es durch Deprivation, Isolation. Folter oder andere gewaltsame Einwirkungen, zum Thema gemacht werden. Zu Hauser vgl. z. B. Hesse (1916) oder weiterführend Struve (1999). 
werden. Diese Erkenntnis ist einerseits ernüchternd, wirft den Menschen quasi auf sich selbst zurück. Andererseits wirkt sie aber auch befreiend, denn sie eröffnet den Weg zur begrifflichen Fassung der eigentlichen menschlichen Freiheit, wie sie bereits Friedrich Engels im "Antidühring" in Übernahme der Argumentation Hegels als Einsicht in die Notwendigkeit und Beherrschbarkeit natürlicher und gesellschaftlicher Prozesse definierte: „Nicht in der geträumten Unabhängigkeit von den Naturgesetzen liegt die Freiheit, sondern in der Erkenntnis dieser Gesetze, und in der damit gegebnen Möglichkeit, sie planmäßig zu bestimmten Zwecken wirken zu lassen“ (Engels 1877-1878, 106).

\section{Zusammenhangsdenken versus Reduktionismus}

Da die das Individuum umgebenden Bedeutungskonstellationen somit nur mehr einen Möglichkeitsraum für sein individuelles Handeln bilden und es zu ihnen in einer prinzipiellen Möglichkeitsbeziehung steht, in der es konkret jeweils so, aber auch anders handeln kann, ist es also nicht mehr nur den Bedingungen unterworfen. Vielmehr kann es über diese nach Maßgabe der je historisch vorhandenen gesellschaftlichen Mittel ein Stück weit verfügen. Damit kann dieser Prozess prinzipiell nicht mehr angemessen durch Konzepte abgebildet werden, die auf veraltete Ansätze in Psychologie (Maiers 2019, 134f.) und Soziologie zurückgreifen, die sich nicht vom oben erwähnten Unmittelbarkeitspostulat gelöst haben und sich weiterhin direkt oder indirekt am Stimulus-Response-Modell oder anderen behavioristischen Vorstellungen orientieren. Der Mensch wird dort nicht auf seiner gesellschaftlich-historischen, sondern lediglich auf seiner tierisch-vorgesellschaftlichen Stufe betrachtet, was sich beispielsweise an den zahlreichen aus Tierexperimenten gewonnenen Aussagen in oft umstandsloser Übertragung auf menschliches Verhalten zeigt. Eine weitere Variante der Reduzierung der gesellschaftlichen Natur des Menschen ist die (ursprünglich im Kognitivismus entwickelte) Variante der Gleichsetzung menschlicher Funktionsweisen mit denen eines Computers. Ich selbst habe zeitweise im Unterricht zu Illustrationszwecken gern auf Shannon und Weavers (1963) eingängiges mathematisches Wirkungsmodell von Kommunikation zurückgegriffen, bis mir die Problematik der grundsätzlichen Unangemessenheit des Modells für soziale Kommunikationsprozesse klar wurde. Allerdings war das meinem damals verkürzten 
Denken geschuldet und nicht den Autoren anzulasten. Denn Shannon und Weaver hatten das Modell primär für technische Zusammenhänge konzipiert, vor allem um „Rauschen“ und Informationsverlust während des Übertragungsprozesses anschaulich zu machen. Dass sie sich in ihrer natur- und technikwissenschaftlichen Herangehensweise dabei im Denken des "Newtonschen Weltbilds" bewegen, ist durchaus angemessen, soweit sie nicht, ihre Fächer überschreitend, sozialwissenschaftliche Schlussfolgerungen ziehen.

Natürlich gibt es in der Kommunikationswissenschaft inzwischen eine differenzierte qualitative Forschung, gibt es mit den Cultural Studies und anderen Konzepten Ansätze, die längst behaviouristisches Denken zurückgedrängt haben. Doch scheint mir die quantitative Methodik mit Orientierung an Stimulus-Response-Modellen in vielen Kernbereichen der Medienforschung wie der Labor- und Umfrageforschung nach wie vor nicht obsolet, gerade also in den Bereichen des subjektiven Handelns, um die es hier geht (z. B. bei Messung und Interpretation von Einschaltquoten im Fernsehen oder der Meinungsforschung durch Umfragen), sodass eine Auseinandersetzung mit diesen notwendig bleibt. Quantitative Forschung hat ihren notwendigen, da unersetzlichen Platz bei Forschungsfragen in gesellschaftlicher, ökonomischer, technischer oder überindividueller Dimension. Aber die nach wie vor noch übliche Reduktion auch bei subjekttheoretischen Fragestellungen auf ein quasi-naturwissenschaftliches Vorgehen als der vermeintlich ,härteren' Methodik, die sich faktisch doch an unterschiedlichen behavioristischen Ansätzen etwa eines Skinner oder Pawlow orientiert, wirkt zunehmend eigenartig. Denn nicht nur in der Psychologie ist wie gezeigt ein solcher Reduktionismus umstritten. Selbst die Naturwissenschaften, aus denen diese Denkweise ursprünglich übernommen wurde, setzen sich seit Längerem deutlich gegen einen derartigen Reduktionismus zur Wehr. Noch scheint kaum zur Kenntnis genommen worden zu sein, dass etwa ein Werner Heisenberg, einer der Schöpfer der Quantenmechanik und der Unbestimmtheitsrelation und wahrlich kein ,Weichling' in Bezug auf physikalische Methodik, in seiner Abhandlung "Ordnung der Wirklichkeit“ (1989, 99ff. und 117) das Grundproblem der Abgrenzung von Quanten- und klassischer Physik, Chemie, Biologie und menschlichem Leben in Anlehnung an Niels Bohr inzwischen präzise auf den Punkt gebracht hat. Er verwendet in diesem Zusammenhang den Begriff der je höheren Organisationsformen, die nicht auf niedrigere (physikalische) reduzierbar seien und meint mit den 
höheren die Lebens- und Sozialwissenschaften. Besonders prononciert formuliert diese Kritik am Reduktionismus auch der Physiknobelpreisträger Robert B. Laughlin: „Die Natur wird nicht allein durch eine Grundlage von Gesetzen auf mikroskopischer Ebene gesteuert, sondern durch starke und allgemeine Ordnungsprinzipien [...]. Was wir sehen, ist eine Veränderung der Weltsicht, in deren Verlauf das Ziel, die Natur durch Zerlegung in immer kleinere Teile zu verstehen, durch das Ziel ersetzt wird, dass man versteht, wie die Natur sich selbst organisiert" (Laughlin 2007, 25). Aus dieser "self-organization of matter", die der Chemienobelpreisträger und Philosoph Ilya Prigogine bereits in den 1980er Jahren analysierte (vgl. Schenkel 1988, 15f.), bildet sich ein neues „physikalisches Ordnungsprinzip“, das auch mit dem Terminus der "Emergenz" (Laughlin) bezeichnet wird.

Bemerkenswert: Es war eine Entdeckung des deutschen Nobelpreisträgers Klaus von Klitzing, die letztlich für diesen Wendepunkt im physikalischen Weltverständnis sorgte (vgl. Laughlin 2007, 124). Durch die Fixierung auf das ,Newtonsche Weltbild' der Physik als der vermeintlich grundlegenderen Wissenschaft ist vielen Sozial- und Kommunikationswissenschaftlerinnen und Kommunikationswissenschaftlern diese fundamentale Umorientierung in der Physik offenbar entgangen, wenn sie nach wie vor nur deren "hartes" methodisches Vorgehen als alleinigen Garanten für die Wissenschaftlichkeit ihrer quantitativen Empirie gelten lassen wollen. $\mathrm{Zu}$ den weiteren problematischen Implikationen, die mit dieser Art Reduktionismus im Medienbereich verbunden sind, habe ich mich bereits an anderer Stelle ausführlicher geäußert (Schenkel 2019), worauf ich hier noch zurückgreifen werde.

Es sollte klar geworden sein, dass damit auch eine marxistische Ideologiekritik nicht umhin kommt, sich ganz praktisch mit dem konkreten Verhalten ${ }^{6}$ menschlicher Individuen zu gesellschaftlichen Denk- und Sprachformen, den damit übermittelten Inhalten und ihrer tatsächlichen Nutzung bei der individuellen Lebensbewältigung dann unter subjekttheoretischem Blickwinkel auseinanderzusetzen statt beispielsweise darauf zu vertrauen, dass dies Alles in der „Kritik der Politischen Ökono-

6 Die Kritische Psychologie hat einen ganz anderen Verhaltensbegriff als behaviouristisch gefärbte Ansätze. Er ist immer als „Verhalten $\underline{\mathrm{Zu}}$ “ gesellschaftlichen Handlungsmöglichkeiten definiert, also aktiv eingreifend im Gegensatz zum "Verhalten unte" Bedingungen, siehe Holzkamp 1983, 277 ff. 
mie" schon objektiv auf den Punkt gebracht sei oder aber andererseits zu meinen, diese einfach um die Freudsche Psychoanalyse zu ergänzen. Eine gängige Fehlinterpretation der sechsten Feuerbachthese von Karl Marx, die er 1845/46 in seiner gemeinsam mit Friedrich Engels verfassten Schrift „Die Deutsche Ideologie“ veröffentlicht hatte, mit der folgenschweren Verkürzung des menschlichen Individuums als „Ensemble menschlicher Verhältnisse", hat auch in kritischen wie marxistisch argumentierenden Ansätzen eine solche Vorstellung der weitgehenden Determiniertheit subjektiven Handelns durch die objektiven Verhältnisse befördert, z. B. in diversen Manipulationstheorien, denen selbst der eigentlich differenziert argumentierende Adorno mit grob verabsolutierenden Formulierungen die eingängigen Stichworte geliefert hat (Horkheimer und Adorno $1997 / 98,141 \mathrm{ff}.)^{7}$

Wir werden sehen, dass solche Verkürzungen beziehungsweise „Ergänzungen“ vor dem Hintergrund kritisch-psychologischer Konzepte nicht mehr haltbar sind.

Aus marxistischer Perspektive, wie sie etwa Horst Holzer und Burkhard Hoffmann in der Medientheorie und Klaus Holzkamp in der Kritischen Psychologie verkörpern, versteht sich eigentlich eine „antireduktionistische" Positionierung quasi von selbst. Denn für Marx und Engels war die Einheit von Natur und Gesellschaft in Gegensatz zu Hegel, aber auch zu den von ihnen etwas abschätzig bezeichneten "Vulgärmaterialisten“, eine aus der Logik und den Forschungsergebnissen von Naturwissen-

7 Marx hatte in der 6. Feuerbach-These geschrieben: „Feuerbach löst das religiöse Wesen in das menschliche Wesen auf. Aber das menschliche Wesen ist kein dem einzelnen Individuum innewohnendes Abstraktum. In seiner Wirklichkeit ist es das ensemble der gesellschaftlichen Verhältnisse. [...] Feuerbach sieht daher nicht, daß das „religiöse Gemüt" selbst ein gesellschaftliches Produkt ist und daß das abstrakte Individuum, das er analysiert, einer bestimmten Gesellschaftsform angehört" (Marx 1845, 6.). Eine marxistisch orientierte Subjektheorie muss hingegen, wie schon im zweiten Abschnitt dargelegt, begreiflich machen, „wie menschliche Naturwesen die Fähigkeits- und Bedürfnisdispositionen entwickeln konnten, sich an der Schaffung und Veränderung gesellschaftlicher Lebensverhältnisse zu beteiligen", sonst laufe man Gefahr, "in einen theoretischen Objektivismus zu verfallen: sei es, dass in einer Art soziologistischen Reduktionismus die Individuen zu bloßen Schnittpunkten gesellschaftlicher Daseinsumstände degradiert, ihre sinnlich-stoffliche Existenz und darin begründeten subjektiven Lebensnotwendigkeiten also weggeleugnet (und unter der Hand und gegen alle erklärte Absicht ideologisch orientierten, wissenschaftlich beschränkten naturalistischen Theoriebildungen überlassen) werden“, so Wolfgang Maiers $(2019,4)$. 
schaften und übergreifender Evolutionstheorie sich ergebende zwingende Grundlage ihrer ,logischen und historischen Methode'. Bei dieser Methode handelt es sich nicht, wie manche Kritiker, die Marx und Engels offenbar gar nicht im Original kennen, unterstellen, um einen besonders raffinierten geistigen Taschenspielertrick. Vielmehr formulierte Friedrich Engels den wesentlichen Grundgedanken dieses Forschungsprinzips in seiner naturwissenschaftlich orientierten und in den Jahren 1873 bis 1886 entstandenen Schrift "Dialektik der Natur" so: "Jetzt auch die ganze Natur in Geschichte aufgelöst, und die Geschichte nur als Entwicklungsprozeß selbstbewußter Organismen von der Geschichte der Natur verschieden" (MEW 20, 504) ${ }^{8}$. Insofern muss man wie Kedrow und Kröber dieses Werk "als eine Art, VorKapital' auffassen, als ein naturwissenschaftliches Heranführen an das Marxsche ,Kapital“" (zit. nach Maiers 2019, 143).

Interessanterweise wird auch in den Kommunikations- und Medienwissenschaften seit Jahrzehnten immer wieder ein entsprechendes übergreifendes Zusammenhangsdenken angemahnt, früh etwa von Gerhard Maletzke oder Klaus Merten (vgl. Schenkel 1988, 183 ff. und 200 ff.), neuerdings wieder von Autoren wie Friedrich Krotz (zuletzt 2018, 36 und 41), freilich bis heute ohne große Konsequenzen. Die Gründe hierfür liegen weniger in persönlichen Defiziten, was ja bei wechselnden Wissenschaftlern und Wissenschaftlerinnen über diesen langen Zeitraum hinweg verwunderlich wäre, sondern in einem nach wie vor grundlegend $\mathrm{zu}$ beschränkten theoretischen und methodischen Horizont. Aber auch historisch-materialistische Ansätze jener Zeit waren nicht frei von bestimmten Verkürzungen (Schenkel 1988, 255 ff.).

Zusammenhangsdenken in der Kommunikationswissenschaft bedeutet, über die Jetztzeit hinaus natur- wie gesellschaftshistorische Wurzeln und historisch-gesellschaftliche Entwicklungen von Medien und Kommunikation im Gesamtzusammenhang zu analysieren, damit Natur-, Gesellschafts- und Individualgeschichte als eine komplexe Einheit zu fassen, was von der Kritischen Psychologie in Anlehnung an Leontjew für die Sozialwissenschaft Psychologie paradigmatisch konkretisiert worden war. Dies ist der Grund, warum ich in diesem Beitrag speziell auf deren

8 Detailliert zur logischen und historischen Methode siehe Engels in seiner Rezension von „Karl Marx Kritik der Politischen Ökonomie“ in der Zeitschrift „Das Volk“ vom 20.08.1859 (MEW 13, 474 ff.) sowie dann auf heute bezogen Holzkamp (2015, 98-181) und Maiers (1999, 1134-1140). 
Ergebnisse rekurriere (vgl. Maiers 1985). Dies bedeutet eben aber auch, die phylogenetischen wie anthropogenetischen Grundlagen menschlicher Kommunikation methodisch mit einzubeziehen. Auch nach dem Umschlag von Natur- in Gesellschaftsgeschichte bleibt der Mensch wie erwähnt ein Teil der Natur, wobei sich in der evolutionären Entwicklung während der Anthropogenese seine Natur zu seiner gesellschaftlichen Natur als neue Qualität biologischer Ausstattung herausgebildet hat (vgl. Holzkamp 1983, 178ff. und Maiers 2019). ${ }^{9}$ Der Jetztmensch hat dadurch nicht nur die Fähigkeit, sondern auch den Zwang zur Gesellschaftlichkeit buchstäblich in seinen Genen. Der nur scheinbar absolute Gegensatz von Mensch und Natur, wie er sich etwa beim Klimawandel zuspitzt, kann nur deshalb in der öffentlichen Diskussion als ein äußerer und absoluter erscheinen, weil die durch die verselbständigten konkret-historischen kapitalistischen Verwertungszwänge der Energiekonzerne verursachten Verkehrungen in der Vorstellungswelt dies so erscheinen lassen und für das Alltagsdenken auseinanderreißen, Täuschungen, deren Wurzeln Marx und Engels schon im ersten Band des Kapital (1864, 86f.) als zwangläufig aus den Verkehrtheiten der Wertform entstehenden „Warenfetisch“, beziehungsweise - bei voller Entfaltung des Kapitals - im dritten Band $(1894,412)$ als „Kapitalfetisch“ analysiert haben. Er ist damit letztlich keine "naturgegebene“ Problematik, wie dies die Alltagssprache nahelegt, sondern eine gesellschaftlich-soziale, durch Profitmaximierung verursachte Problematik, die ohne gravierende Eingriffe auch auf dieser Ebene nicht gelöst werden kann.

\section{Individuelle Kommunikation als Teil der gesellschaftlichen Natur des Menschen}

Wenn jenseits der Einseitigkeiten einer rein naturevolutionistischen oder aber andererseits einer rein soziologischen Sicht die biologisch verankerte gesellschaftliche Natur des Menschen mit ihrem Teilaspekt des auf Medien bezogenen Handelns in einer einheitlichen Gesamtsicht erfasst

9 Vgl. nochmals Friedrich Engels 1873-1878 in Dialektik der Natur (MEW 20, 317ff.) sowie Holzkamp (1984, 2f.). Zu den gravierenden methodologischen Konsequenzen für die Sozialwissenschaften vgl. Maiers (1985, 119ff.). 
werden soll, muss der wirkliche Zusammenhang dieser Ebenen wissenschaftlich nachvollziehbar werden.

Was bedeutet das nun für menschliche Kommunikation und darin eingebettet die Kommunikation von konkreten Individuen im Spannungsfeld von persönlicher Kommunikation und Mediennutzung? Selbstverständlich ist ihre Entwicklung in diesen evolutionären Prozess eingebettet und nicht Ergebnis einer wie auch immer gearteten Emanation höherer Mächte, wie es das bekannte Zitat aus dem Johannesevangelium: „Im Anfang war das Wort“ unterstellt (Württembergische Landesbibliothek Stuttgart 2019). Vielmehr sind die kommunikativen und ,medialen' Aspekte menschlichen Daseins in einem natürlichen Entwicklungsprozess bereits weit vor der Durchsetzung menschlicher Gesellschaftlichkeit in einem Jahrmillionen währenden evolutionären Prozess und nicht plötzlich entstanden. Schon früh haben sich mit dem Entstehen von Information und deren Übermittlung zunächst Frühformen physischer Träger,Medien' herausgebildet, die dann zu physikalischen und biologischen 'Mittlern' der entstehenden Kommunikation von Lebewesen wurden. Jenseits kommunikations- und medienwissenschaftlicher Fragestellungen sind Information und deren Träger ja auch Gegenstand anderer Einzelwissenschaften wie der Kosmologie, der Teilchenphysik, der Astronomie, der Informationswissenschaft, der Informatik, der Geologie, der Naturevolution oder der Biologie (Phylogenese des Lebens und Tierkommunikation) geworden, lange bevor sich die Kommunikationswissenschaft als eigenständige Fachdisziplin herausgebildet hat - einer der Gründe für die Kommunikationswissenschaften, sich als interdisziplinär zu definieren.

Ein lediglich bausteinmäßiges Zusammenfügen unterschiedlicher Erkenntnisse aus verschiedenen Wissenschaftszweigen ohne wissenschaftstheoretische Vermittlung, wie wir das in kommunikationswissenschaftlicher Literatur öfters vorfinden ${ }^{10}$, birgt die Gefahr eines Eklektizismus ohne zusätzlichen Erkenntnisgewinn oder - weit gefährlicher - von Fehleinschätzungen und des Übersehens wichtiger Zusammenhänge. Weisen

10 Leider sind dieser Versuchung selbst sonst sehr akkurat argumentierende Autoren wie Klaus Merten erlegen, als er einmal meinte, die Heisenbergsche Unbestimmtheitsrelation zur Klärung menschlicher Kommunikation/Wirkungen mit heranziehen zu können, was aber Heisenbergs Gesamtdenken völlig widerspricht (Merten 1982, 41). Natürlich haben wir heute aber einen besseren Überblick über die verschiedenen Qualitäten dieser Ebenen als vor 40 Jahren. 
vorfindliche Phänomene der Wirklichkeit wie gegenseitiger Informationsaustausch - er ist ja eines der Kriterien für Kommunikation - auf Gemeinsamkeiten in unterschiedlichen Wissenschaftsgegenständen hin, muss über die Aneinanderreihung der bloßen Forschungsergebnisse der unterschiedlichen wissenschaftlichen Ansätze hinaus eine wissenschaftstheoretische Vermittlung ihrer jeweils zugrundeliegenden Theorien geleistet werden. Es ist also zum Beispiel zu prüfen, ob sie überhaupt von einer gleichen Definition von "Kommunikation" ausgehen, ferner sind qualitative Übergänge von einer Ebene zur anderen wissenschaftlich abzubilden, Geltungsbedingungen abzugleichen und anderes mehr.

Individuelle und generell menschliche Kommunikation sind also von ihrer Entstehung an ein Teilaspekt der naturhistorischen Prozesse. Menschliche Kultur und Kommunikation sind keineswegs erst späte Zutat zur naturevolutionären und anthropogenetischen sowie dann gesellschaftlich-historischen Entwicklung, im Gegenteil: Studien zur Kommunikations-, Symbol- und Zeichenentstehung haben gezeigt, dass diese zu fassen sind als „,kulturelle und darin einbegriffene kommunikative virtuelle wie reale Aktivitäten [...], gleichursprüngliche Wesenheiten menschlicher Entwicklung [...], die ihre Wurzeln bereits in der vormenschlichen Evolution haben" (Schenkel 2017, 101ff.) und so sogar zur mächtigen Triebkraft der Anthropogenese hin zu einem gesellschaftlich-historischen System wurden .

Schon während ihrer naturhistorischen Herausbildung auf tierischer Ebene hat sich Kommunikation als informationsspezifischer Aspekt der tierischen und dann vor- und frühmenschlichen Sozialität verselbständigt, wenngleich sie trotz zunehmender Eigenständigkeit an diese gekoppelt bleibt. Mit Durchsetzung des gesellschaftlich-historischen Prozesses in der menschlichen Lebensweise verändert sich Kommunikation hin zu einer neuen, gesellschaftlichen Qualität, die über die weiterhin wirkenden biologisch-genetischen Grundlagen hinaus zum dominanten Bestimmungsmoment wird: „Die Informationsbeziehungen der naturwüchsigen Sozialität werden zum spezifischen Informationsaspekt der Beziehung Mensch-Geräte-Umwelt und damit einbegriffener innergesellschaftlicher Informationsentwicklung" (ebd.). Kommunikation ist also jetzt zu verstehen als spezifischer Informationsaspekt des menschlichen Sozialen, damit aber nach wie vor nicht auf dieses reduzierbar oder mit diesem gleichzusetzen, wie das noch in manchen Konzepten nach dem Motto soziales Handeln ist gleich Kommunikation geschieht. 
In einer Koevolution von Werkzeug-, Kommunikations- und Symbolverhalten (Maiers 2006, 49) in diesem natur- und dann zunehmend gesellschaftshistorischen Prozess haben menschliche Kommunikation und (Bedeutungs-)Wahrnehmung Spezifika gewonnen, die zu entscheidenden Faktoren für die Stabilisierung der Gattung gegenüber anderen hochentwickelten Organismensozietäten und für die Herausbildung ihrer spezifischen psychischen Fähigkeiten und der dadurch möglichen komplexen Kommunikationsfähigkeit wurden (vgl. auch Holzkamp 1983, 301ff.). Gerade die „symbolische Repräsentation von Umwelttatbeständen und -erfordernissen im Prozess der Aneignungs- und Vergegenständlichungsaktivitäten ermöglichte eine raumzeitlich und Generationen überdauernde Weitergabe von Informationen und Fertigkeiten als mächtige Grundlage gesellschaftshistorischer Entwicklung" (Schenkel 2017, 103f.). In gewisser Weise wäre das schon ein evolutionär unterfüttertes ganz früh ansetzendes Argument für die außerordentliche Bedeutung von Medien, die inzwischen unter dem Begriff der Medialisierung als abhebbarer eigenständiger Gegenstand in der Kommunikationstheorie thematisiert wird (vgl. Schenkel 2019). Denn Kommunikation benötigt gleichursprünglich ein Medium als Träger ihres Informationsaustausches. In frühen Stadien der Tierkommunikation waren das zunächst körpereigene Teile wie der Fuß des stampfenden Elefanten, der dadurch mittels des Bodens - ebenfalls ein Medium - tieffrequent über weite Entfernungen kommunizieren kann oder der Brustkorb des Primaten, den dieser aus Imponiergehabe zum Trommeln benutzt - anschauliche Beispiele übrigens, wie ursprünglich motorische Fähigkeiten für zusätzliche kommunikative Zwecke umfunktioniert werden (vgl. dazu Schurig 1975 Band 1, 179, 185). Die auf tierischer Ebene noch an körperliche und natürliche Umgebungsmomente gebundenen Aktivitäten entwickeln sich dann im Verlauf der anthropogenen Entwicklung zu Aneignungs- und Vergegenständlichungsaktivitäten mit Werkzeugen und veränderten Umgebungsqualitäten weiter, die dann als Medien im eigentlichen Sinne bezeichnet werden.

Auf gegenwärtigem weit fortgeschrittenen Niveau spätkapitalistischer Warenproduktion sind nicht nur die technisch höchst elaborierten medialen Instrumente für netzbasierte Kommunikationsmöglichkeiten, die ganz im Sinne des etwas nonchalant argumentierenden Marshal McLuhan (McLuhan und Powers 1995) als Erweiterungen des menschlichen Körpers verstanden werden können, als neuer Wirkfaktor einzubeziehen, sondern auch die spezifischen Verwertungsimperative des 
Kapitals und auch die durch Digitalisierungsprozesse gegebenen Modifizierungen (vgl. Sevignani 2018; Fuchs und Sevignani 2013) in Rechnung zu stellen, die natürlich ebenfalls die Voraussetzungen der prinzipiellen Möglichkeitsbeziehung menschlicher Subjekte tangieren.

Nicht nur aus marxistischer Perspektive sind es neben den gesellschaftlich-formationsspezifischen Grundlagen bürgerlicher Ideologieproduktion wie Waren- bzw. Monopolfetisch, machtinduzierter politischer Kommunikation, Öffentlichkeitsarbeit und PR vor allem die individuell je unterschiedlichen Verwendungszusammenhänge (Rezeptionsweisen) von Kommunikaten, die die konkreten Wirkungsweisen von Ideologie ermöglichen und die es zu entschlüsseln gilt. Übrigens findet sich diese Sichtweise auch in neueren Versuchen, personalisierter' netzbasierter Zielgruppenansprachen im Marketing, das sich weit entfernt wähnt von kritischen oder gar marxistischen Perspektiven. Die mir im Umfeld dieses Beitrages zu Bedenken gegebene interessante Frage, ob solche Phänomene als kapitalistisch überformte Subjektaspekte interpretierbar sind oder diese im Gegenteil gerade unterlaufen, müsste sicher genauer untersucht werden.

\section{Restriktive Handlungsfähigkeit und deutendes Denken}

Die verkürzende Sicht des Unmittelbarkeitspostulats und des StimulusResponse-Modells, das übergeordnete gesellschaftliche Imperative meist nicht als Variable in Laborversuchen abbildet, bedeutet noch lange nicht, dass menschliches Handeln tatsächlich von den produktivkraftbedingten, je gesellschaftlich-historisch unterschiedlichen Rahmenbedingungen ohne Verlust seiner Wesensbestimmungen isolierbar ist. Bei genauem Hinsehen schlagen diese Rahmenbedingungen oft doch als Faktoren in Untersuchungsdesigns durch und müssen dann als ,Stör'-faktoren mühsam wieder weginterpretiert werden. ${ }^{11}$ Gesellschaftliche Zwänge führen im Gegenteil - hier auf spät- oder monopolkapitalistisch geprägte

11 Vgl. dazu die fundamentale wissenschaftstheoretische Kritik an dieser Art von Wissensgewinnung der Experimentalpsychologie durch Holzkamp (1964) und zusammengefasst in Holzkamp (1972). 
Lebenszusammenhänge fokussiert - dazu, dass sich die Möglichkeitsbeziehung des Individuums in zwei Richtungen ihrer personalen Handlungsfähigkeit entwickelt, die im kritisch-psychologischen Verständnis als restriktive und verallgemeinerte Handlungsfähigkeit bezeichnet werden. Ihre Entgegensetzung ist nur relativ zu verstehen als unterschiedliche Aspekte der Handlungsfähigkeit, also nicht im Sinne von verselbständigten Handlungsweisen (Holzkamp 1983, 374ff.). Fundiert ist diese zwieschlächtige Handlungsfähigkeit mit jeweils unterschiedlichen emotionalen und kognitiven Befindlichkeiten, so einem entsprechenden Denkvermögen zwischen seinen beiden Polen des deutenden und begreifenden Denkens, die wiederum für den Ideologieaspekt von herausgehobener Bedeutung sind. Das von Holzkamp „deutend“ genannte Denken im Rahmen restriktiver Handlungsfähigkeit ist gekennzeichnet als ein verkürztes Denken, indem eine real mögliche Verfügungserweiterung unbewusst gehalten werden muss und das Individuum sich auf das kognitive Erfassen von „Faktizitäten“ beschränkt. Dies ist dann verknüpft mit einer Reduzierung auf Muster wie „Privatheit" und auf die bereits genannte Unmittelbarkeitsverhaftetheit, so als ob Probleme nur individuell oder interaktiv lösbar wären, wobei dann der ja trotzdem objektiv in den umgebenden Bedeutungsstrukturen vorhandene gesamtgesellschaftliche Verweisungszusammenhang ausgeblendet wird (Holzkamp 1983, 388, Leontjew 1977, 241ff.). Dies charakterisiert die Kritische Psychologie in kritischer Aufhebung Freudscher Erkenntnisse als Verdrängungsprozess (Holzkamp 1984, 15ff.). Damit findet dann zum Beispiel keine Unterscheidung mehr statt zwischen Erscheinung und Wesen vorhandener Gegebenheiten, die ja oft nicht zusammenfallen. In Bezug auf Andere wird dann die Möglichkeit des gemeinsamen Bezugs auf allgemeinwohlorientierte Ziele im kooperativen Handeln ausgeklammert, bleiben lediglich persönliche, ,instrumentelle' Beziehungen, übrig, in denen das Gegenüber nur noch unter dem Aspekt der Nützlichkeit für die eigenen Partikularinteressen betrachtet wird.

\section{6 "Subkutane“ Ideologiebildung als Rezeptionsangebot}

Zur Nutzung angeboten werden solche Muster - und damit sind wir wieder beim Thema Ideologieproduktion und -verarbeitung - in vielen 
gängigen Filmen sowohl der Kinoproduktion als auch der privaten und leider oft - entgegen ihrem Programmauftrag - der öffentlich-rechtlichen Sendeanstalten. Dadurch entsteht gleichsam eine ,subkutane' Ideologieproduktion zur Stabilisierung der Herrschaftsverhältnisse, ohne dass sie als solche sofort unmittelbar empfunden oder erkennbar würde. Gerade das erhöht den Grad ihrer Wirksamkeit. In der Regel kann erst eine differenziert Film- und Fernsehanalyse solche Muster sichtbar machen, für die aber auf den einschlägigen Fernsehseiten der Presse leider immer weniger Platz eingeräumt wird.

Als konkretes Beispiel für subkutane Ideologiebildung, das für viele stehen kann, nehme ich den in der ARD am 15.05.2018 zur Hauptsendezeit ausgestrahlten Fernsehfilm „13 Uhr mittags“. Dieser ist als Persiflage angelegt auf den legendären preisgekrönten Western "12 Uhr mittags“ („High Noon“) von Fred Zinnemann - mit Gary Cooper und Grace Kelly in den Hauptrollen -, sehr gut inszeniert und mit dem prominenten Schauspieler Jörg Schüttauf in der Hauptrolle besetzt. Tatsächlich verkehrt er aber - und darin liegt hier die Raffinesse der Ideologiebildung die humanistische Botschaft des Ursprungsfilms in das völlige Gegenteil einer individuell-utilitaristischen Sichtweise. Im historischen Film kämpft bekanntlich ein moralisch integrer Marshall (verkörpert von Gary Cooper) vergeblich gegen die angepasste Doppelmoral der Kleinbürger seiner Stadt an. Sie lassen ihn im entscheidenden Augenblick im Stich und wollen ihn erst wieder nach seinem überraschenden Erfolg gegen eine kriminelle Bande anstellen, was er dann dankend ablehnt. Das war eine für die Zeit seiner Entstehung 1952 erstaunliche Kritik an den inneren USVerhältnissen, die dem Film und seinem Regisseur prompt landesweit Boykottaufrufe und den Vorwurf „unpatriotischen“ Verhaltens etwa durch den reaktionären „Law and Order"-Schauspieler John Wayne eintrugen.

In dem Fernsehfilm „13 Uhr mittags“ hingegen verkehrt sich diese inhaltliche Stoßrichtung des Plots ins Gegenteil: Hier wird ein korrupter Polizist, der durch seine Falschaussage einen Dieb so ins Gefängnis bringt, dass er sich dessen verschwundene Beute später unter den Nagel reißen kann, auf witzige Weise zum sympathischen und in seinen Handlungen letztlich versteh- und nachahmenswerten Helden stilisiert, gut illustrierend, was deutendes Denken und unmittelbares Verhaftetheitbleiben in den Verhältnissen meint. Klare Botschaft: Sei clever, verfolge deinen persönlichen Vorteil, ist doch nichts dabei, das schadet ja der Öffentlichkeit 
nicht; also ein gut verpacktes ideologisches Angebot, sich doch mit den Verhältnissen zufrieden zu geben und persönlich schadlos zu halten, um in ihnen glücklich werden zu können, ohne sie konfliktreich verändern zu müssen.

Derartige subkutan verabreichte Muster häufen sich seit einigen Jahren auffällig auch in öffentlich-rechtlichen Produkten, zum Beispiel in den sonntäglichen, zunehmend nur noch persönliche Beziehungstaten thematisierenden Tatorten wie kürzlich im Dresdner ARD-Tatort „Nemesis“ am 18.08.2019. Verweise auf mafiöse Strukturen und systematische Geldwäsche dienen hier letztlich nur noch als Staffage für individuelle Mordgeschichten. Sicher wäre es eine noch tiefer gehende Analyse wert, inwieweit dies lediglich das Durchschlagen einer blinden, der bürgerlichen Oberfläche verhafteten Sichtweise der Drehbuchautor*innen und Programmverantwortlichen durch deutendes Denken ganz im Sinne des Marxschen Kapitalfetischs oder doch gezielteren Absichten geschuldet ist. ${ }^{12}$

Die damit einhergehende Verinnerlichung und Psychisierung gesellschaftlicher Widersprüche durch Verdrängung und Abschieben des Gewussten als Unbewusstes bleibt wie gesagt brüchig, da Verdrängung, ein dynamischer Prozess ist, sich diese Variante der Handlungsfähigkeitssicherung stets durchsetzen muss gegen die in der je umgebenden

12 Leider ist eher Letzteres anzunehmen, scheint diese Programmpolitik nicht dem Zufall geschuldet, sondern vorsätzlich zu sein. Denn gleichzeitig werden qualitativ hervorragende und gleichwohl unterhaltsame Sendungen wie etwa die sechsteilige Serie „Die Neue Zeit“ über die Entwicklung des Bauhauses mit einem Schwerpunkt auf dessen heiklen Umgang mit Frauen im ZDF oder kritische Satiresendungen nicht etwa um 20.15 Uhr sondern nur zu nachtschlafender Zeit nach 22 Uhr gezeigt, obwohl die Gebühren finanzierten ARD und ZDF keinerlei Zwang zur Einschaltquote unterworfen sind wie die sich darüber finanzierenden Privatsender. Sie verstoßen damit massiv gegen ihren in den Rundfunkstaatsverträgen formulierten quasiöffentlichen Auftrag, nach dem sie nicht nur eine freie „individuelle und öffentliche Meinungsbildung fördern und ein umfassendes Bild der deutschen Wirklichkeit" vermitteln (ZDF 2018, § 5), sondern damit ,, auch die demokratischen, sozialen und kulturellen Bedürfnisse der Gesellschaft" erfüllen sollen, indem sie "Beiträge insbesondere zur Kultur anzubieten" haben (Medienanstalten 2019, § 11). Dieses Programm-Missmangement bietet fahrlässig eine weitere offene Flanke für immer wiederkehrende Forderungen nach ihrer Verkleinerung beziehungsweise Privatisierung, die inzwischen nicht nur von CSU und AfD erhoben werden. Denn wenn sie sich nicht mehr in Qualität und Angebotsspektrum von privaten Sendeanstalten oder Streaming-Portalen unterscheiden, wird ihrer Existenz langfristig der Boden entzogen 
Bedeutungskonstellation ebenfalls vorhandenen und damit auch der menschlichen Wahrnehmung zugänglich bleibenden Denkmöglichkeiten verallgemeinerter Handlungsfähigkeit (Holzkamp-Osterkamp 1976, 192ff.). Dies macht die gesamte Persönlichkeit brüchig und widersprüchlich und kann, da sich der real existierende Zusammenhang nicht völlig verdrängen lässt, zu einer Selbstfeindschaft (Osterkamp) führen mit allen Konsequenzen der psychischen Nicht-Bewältigung und Symptomen bis hin zur psychischen Krankheit oder Sucht. Denn Anpassungsverhalten dient zwar im Augenblick tatsächlich der kurzfristigen Absicherung der eigenen Handlungs- und Lebensfähigkeit, untergräbt aber damit deren langfristige Absicherung, die nur durch Änderung der Verhältnisse und den Kampf dafür herbeigeführt werden kann (vgl. Holzkamp 1983, 377ff.).

\section{Ausblick: Individualkommunikation und Kommunikationscharaktere}

Die unterschiedliche Nutzung von Medien bei der Sicherung individueller Handlungsfähigkeit ist damit notwendig ebenfalls zwiespältiger Natur, die bei jeder Analyse zu berücksichtigen ist. Hinzu kommt, dass durch die aktuellen medialen Entwicklungen neue Möglichkeitsräume für die Sicherung von Handlungsfähigkeit entstehen. Gegenwärtig sind wir Zeuge einer Verschiebung von klassischer linearer Mediennutzung hin zu einer örtlich und zeitlich ungebundenen, immer stärker non-linearen Nutzung generisch digitaler Angebote und dies zunehmend auf mobile Weise (Frees, Kupferschmidt und Müller 2019, 326ff., Engel, Maier und Müller 2018, 330), womit die individuellen Nutzungsmuster noch persönlicher und vielfältiger werden können. Wie aber werden diese vor dem Hintergrund des fortschreitenden historischen Vergesellschaftungsprozesses und seiner Realisierung über politökonomische Verwertungszwänge der Medienkonzerne noch sozialwissenschaftlich erschließbar, ohne eine Reduktion der Individuen auf ihre statistisch erfassbaren Eigenschaften, die das nicht wissenschaftlich abbilden kann? Bedingt durch die sich ausdehnende Medialisierung der Verhältnisse gewinnt die individuelle Mediennutzung zwangsläufig eine wachsende Funktionalität für die Sicherung individueller Handlungsfähigkeit. Menschliche Handlungs- 
fähigkeit als generelle Möglichkeitsbeziehung zur gesellschaftlich geschaffenen Umwelt macht die umgebenden gesellschaftlichen Bedeutungsstrukturen zu Prämissen individuellen Handelns, die dieses nicht mehr determinieren wie im klassischen Unmittelbarkeitspostulat unterstellt. Dadurch werden auch die historischen Gegebenheiten nur mehr zu einem mit zu berücksichtigenden Erklärungsfaktor. Da gesellschaftliche Verhältnisse bislang immer Klassenverhältnisse mit gegensätzlichen Interessensgruppen sind, kann sich je nach Klassen- und Schichtenzugehörigkeit individuelle Handlungsfähigkeit aber nur im Spannungsverhältnis zwischen Anpassung und Widerstand gegenüber solchen Verhältnissen realisieren und damit im Ringen um ein spezifisches Verhältnis der eigenen restriktiven beziehungsweise verallgemeinerten Handlungsfähigkeit. Auch die menschliche individuelle Kommunikation und Mediennutzung als zwingender Bestandteil der Sicherung von individueller Handlungsfähigkeit ist damit in ihren restriktiven wie verallgemeinerten Anteilen bei deren Realisierung zu berücksichtigen und zu erforschen. In den je vorgegebenen gesellschaftlichen Kommunikationsverhältnissen haben sich aber produktivkraft- und - im Kapitalismus - verwertungsbedingt spezifische objektive Informationsraster herausgebildet, aktuell z. B. objektive Möglichkeiten wie Mails schreiben, Einweg- oder Streaming-Fernsehen oder Facebook und Instagram nutzen usf., die ich in Anlehnung an Marx als „Kommunikations-Charaktere“ charakterisiert hatte (Schenkel 1988, 270ff. und 306) ${ }^{13}$. Auch zu diesen Kommunikations-Charakteren (= Kommunikationsmustern) als Handlungsmöglichkeiten verhalten sich Individuen aktiv unterschiedlich. Ihre tatsächliche Nutzung als Prämissen für ihr kommunikatives Handeln wird dann ebenfalls anhand der Alternative: restriktive Handlungsfähigkeit als individuell-unmittelbare Bedürfnisbefriedigung oder verallgemeinerte Handlungsfähigkeit als gemeinsame kollektive Erweiterung der gesellschaftlichen Lebensmöglichkeiten untersuchbar. Erst durch einen solchen theoretischen Einbezug der unterschiedlichen Ebenen von Gesellschaft, Sozialität und individuellem Handeln kann eine Verabsolutierung oder Vereinseitigung je einer dieser

13 Friedrich Krotz spricht später in seiner Habilitationsschrift von 2001 unter Bezug auf den Symbolischen Interaktionismus ebenfalls verblüffend ähnlich von Medienkommunikation als Modifikation von interpersonaler Kommunikation und von der Ausbildung eines durch die Kommunikationsumgebung geprägten spezifischen „Kommunikationscharakters" (Krotz 2001, 92), ohne allerdings meine Arbeit von 1988 zu erwähnen. 
Ebenen vermieden werden. Damit wird aber andererseits auch über allgemeine Ideologiekritik hinaus ein Instrument erschließbar, um tatsächliche Rezeptions- und Wirkungsweisen von Ideologie durch konkrete Individuen zu untersuchen. ${ }^{14}$ Insofern kann die Untersuchung individueller Medialkommunikation unter Fruchtbarmachung kritisch-psychologischer Erkenntnisse auch über spezifisch marxistische Ansätze hinaus eine Bereicherung für kritische Kommunikationswissenschaft darstellen.

\section{Literatur}

Engels, Friedrich. 1859. Rezension von ,Karl Marx Kritik der Politischen Ökonomie‘ in der Zeitschrift Das Volk vom 20.8.1859. MEW 13, 474ff.

Engels, Friedrich. 1873-1883. Dialektik der Natur. MEW 20, 317ff.

Engels, Friedrich. 1877-1878. Antidühring. MEW 20, 259ff., 504

Engels, Friedrich. 1845/ 46 (zusammen mit Karl Marx). Die Deutsche Ideologie, MEW 3, $9 \mathrm{ff}$.

Engel, Bernhard, Lothar Mai und Thorsten Müller. 2018. Massenkommunikation Trends 2018: Intermediale Nutzungsportfolios. Media Perspektiven 7-8: 330-347, https://www.ard-werbung.de/fileadmin/user_upload/media-perspektiven/pdf/2018/070818_Engel_Mai_Mueller.pdf. Zugegriffen: 28. November 2019.

Frees, Beate, Thomas Kupferschmidt, und Thorsten Müller. 2019. ARD/ZDF-Massenkommunikation. Trends 2019: Non-lineare Mediennutzung nimmt zu. Media Perspektiven 7-8: 314-333, https://www.ard-werbung.de/fileadmin/user_upload/media-perspektiven/pdf/2019/070819_Frees_Kupferschmitt_Mueller.pdf. Zugegriffen: 28. November 2019.

Fuchs, Christian, und Sebastian Sevignani. 2013. What Is Digital Labour? What Is Digital Work? What's their Difference? And Why Do These Questions Matter for Understanding Social Media? Triple C 11 (2): 237-293, https://triple-c.at/index.php/tripleC/article/view/461. Zugegriffen: 28. November 2019.

Haug, Frigga, Wolfgang Maiers, und Ute Osterkamp. 2015. Klaus Holzkamp Schriften VI. Hamburg: Argument.

Heisenberg, Werner. 1989. Ordnung der Wirklichkeit. München/Zürich: Piper.

14 Aus einem subjektwissenschaftlichen Anspruch heraus erfordert dies eine andere empirische Methodik, die grundsätzlich über die als kontrollwissenschaftlich charakterisierten Methoden hinausgeht (Holzkamp 1983, 522) und von der Kritischen Psychologie inzwischen ansatzweise entwickelt wurde (vgl. etwa Markard 2017, 174ff.). Für die Kommunikationswissenschaften konnte das bislang nicht geleistet werden. Dies bleibt eine große Herausforderung. 
Hesse, Günther. 1916. Kaspar Hauser, das Kind von Europa, der Sohn des Pfarrers und Botanikers Wolfgang Hechenberger aus Tirol, spricht Latein.: Und kommt aus M.L.Ö.!

Hoffmann, Burkhard. 1976. Die Entwicklung einer materialistischen Theorie der Massenkommunikation in der BRD. Argument-Sonderband 10: 306-319.

Holzer, Horst. 1969. Massenkommunikation und Demokratie in der Bundesrepublik Deutschland. Opladen: Leske.

Holzer, Horst. 1977. Referat auf dem 4. Kongress Kritische Psychologie vom 6.-9. Februar 1977 an der FU Berlin, Kongressbericht Band 2.

Holzer, Horst. 1978. Der Gebrauchswert gesellschaftlicher Kommunikation und die Medienwirklichkeit. Manuskript des Gastvortrages im Rahmen des Seminars „Kritische Psychologie und Kommunikationswissenschaft" (Schenkel) am Institut für Publizistik der FU-Berlin, gehalten am 07.07.1978.

Holzer, Horst. 1978. Der ,Gebrauchswert' gesellschaftlicher Kommunikation und die kapitalistische Medienwirklichkeit. Manuskript des Gastvortrages am 07.07.1978 im Rahmen des Seminars „Kritische Psychologie und Kommunikationswissenschaft“ (Schenkel) am Institut für Publizistik der FU-Berlin.

Holzkamp, Klaus. 1964. Theorie und Experiment in der Psychologie. Eine grundlagenkritische Untersuchung, in: Holzkamp, Klaus (2005), Gesammelte Schriften Band II.

Holzkamp, Klaus. 1972. Verborgene anthropologische Voraussetzungen der allgemeinen Psychologie, in: Holzkamp, Klaus: Kritische Psychologie. Vorbereitende Arbeiten, 35-73

Holzkamp, Klaus. 1973. Sinnliche Erkenntnis - Historischer Ursprung und gesellschaftliche Funktion der Wahrnehmung. Hamburg: Argument.

Holzkamp, Klaus. 1977a. Berufsverbot im Öffentlichen Dienst: Wer Indoktriniert wen? Rede, gehalten am 28. Januar 1977 im Rahmen einer Veranstaltung der Aktionsgemeinschaften von Demokraten und Sozialisten (ADSen) der Hochschulen Westberlins „Gegen den Abbau demokratischer Rechte“. In Klaus Holzkamp Schriften VI, herausgegeben von Frigga Haug, Wolfgang Maiers, und Ute Osterkamp (2015). 393ff., Hamburg: Argument.

Holzkamp, Klaus. 1977b. Die kategoriale theoretische Erfassung der Vermittlung zwischen konkreten Individuen und ihren gesellschaftlichen Lebensbedingungen durch die Kritische Psychologie. In Klaus Holzkamp Schriften VI, herausgegeben von Frigga Haug, Wolfgang Maiers, und Ute Osterkamp (2015). 417ff., Hamburg: Argument.

Holzkamp, Klaus. 1982. Einführende Vorbemerkungen in: A. N. Leontjew. 1982. Tätigkeit, Bewußtsein, Persönlichkeit. Köln.

Holzkamp, Klaus. 1983. Grundlegung der Psychologie. Frankfurt am Main/New York: Campus.

Holzkamp, Klaus. 1984. Zum Verhältnis zwischen gesellschaftlichem Prozess und individuellem Lebensprozess. Konsequent Sonderband 6. Streitbarer Materialismus. Berlin (West).

Holzkamp, Klaus. 2015. Die historische Methode des wissenschaftlichen Sozialismus und ihre Verkennung durch J. Bischoff. In Klaus Holzkamp Schriften VI, herausgegeben von Frigga Haug, Wolfgang Maiers, und Ute Osterkamp. Hamburg: Argument. 
Holzkamp-Osterkamp, Ute. 1976. Grundlagen der psychologischen Motivationsforschung 2 - Die Besonderheit menschlicher Bedürfnisse - Problematik und Erkenntnisgehalt der Psychoanalyse. Frankfurt am Main/New York: Campus.

Holzkamp-Osterkamp, Ute. 1983. Ideologismus als Konsequenz des Ökonomismus, Zur Kritik am Projekt Ideologietheorie (PIT). Forum Kritische Psychologie, Nr. 11.

Horkheimer, Max und Theodor Adorno. 1997/98. Aufklärung als Massenbetrug. In Dialektik der Aufklärung, Gesammelte Schriften Band 3. Frankfurt am Main: Suhrkamp.

Hund, Wulf D. 1976. Nachricht und Informationsfetisch. Zur Theorie der gesellschaftlichen Kommunikation. Darmstadt: Luchterhand.

Krotz, Friedrich. 2001. Die Mediatisierung kommunikativen Handelns. Der Wandel von Alltag und Sozialen Beziehungen, Kultur und Gesellschaft durch die Medien. Wiesbaden: Westdeutscher Verlag.

Krotz, Friedrich. 2018. Medienwandel und Mediatisierung. Einstieg und Überblick. In Mediatisierte Gesellschaften. Medienkommunikation und Sozialwelten im Wandel, herausgegeben von Andreas Kalina, Friedrich Krotz, Matthias Rath, und Caroline Roth-Ebner. BadenBaden: Nomos.

Kruse, Norbert, und Manfred Ramme. 1988. Hamburger Ringvorlesung. Kritische Psychologie. Wissenschaftskritik, Kategorien, Anwendungsgebiete.

Laughlin, Robert B. 2007. Abschied von der Weltformel. Die Neuerfindung der Physik. München: Piper.

Leontjew, A.N. 1977. Probleme der Entwicklung des Psychischen. Mit einer Einführung von Klaus Holzkamp und Volker Schurig. Kronberg/Ts.: Athenäum-Verlag.

Markard, Morus. 2017. Kritische Psychologie. Forschung vom Standpunkt des Subjekts. In Handbuch Qualitative Forschung in der Psychologie, herausgegeben von Günter Mey und Katja Mruck. 166ff., Wiesbaden: Springer.

Marx, Karl. 1867. Das Kapital, Erster Band, MEW 23

Marx, Karl. 1885. Das Kapital, Zweiter Band, Hrsg. v. Friedrich Engels, MEW 24

Marx, Karl. 1894. Das Kapital Dritter Band, MEW 25

Marx, Karl, und Friedrich Engels. 1969. Werke (MEW). Band 3, herausgegeben vom Institut für Marxismus-Leninismus beim Zentralkomitee (ZK) der Sozialistischen Einheitspartei Deutschlands (SED). Berlin (DDR): Dietz.

Marx, Karl, und Friedrich Engels. 1981. Werke (MEW). Band 13, herausgegeben vom Institut für Marxismus-Leninismus beim Zentralkomitee (ZK) der Sozialistischen Einheitspartei Deutschlands (SED). Berlin (DDR): Dietz.

Marx, Karl und Engels, Friedrich. 1962. Werke (MEW). Band 20, herausgegeben vom Institut für Marxismus-Leninismus beim Zentralkomitee (ZK) der Sozialistischen Einheitspartei Deutschlands (SED). Berlin (DDR): Dietz.

Marx, Karl. 1845. Thesen über Feuerbach. In Karl Marx und Friedrich Engels MEW 3, 57.

Maiers, Wolfgang. 1988. Sechzig Jahre Krise der Psychologie. Forum Kritische Psychologie $21,23-82$. 
Maiers, Wolfgang. 1985 (1). Methodologische Implikationen des Leontjewschen Tätigkeitskonzepts. Forum Kritische Psychologie 15, 110ff.

Maiers, Wolfgang. 1985 (2). Menschliche Subjektivität und Natur In Forum Kritische Psychologie 15, 1985, herausgegeben von Klaus Holzkamp. Berlin (West): Argument Verlag.

Maiers. Wolfgang, und Morus Markard. 1987. Der Beitrag der Kritischen Psychologie zur Erkennbarkeit und Emanzipation menschlicher Subjektivität, in Kritische Psychologie als Subjektwissenschaft. Klaus Holzkamp zum 60. Geburtstag.

Maiers, Wolfgang. 1988 (a). Sechzig Jahre Krise der Psychologie. Forum Kritische Psychologie 21, 23-82.

Maiers, Wolfgang. 1988 (b). Problemgeschichte der Kritischen Psychologie, in: Kruse, Norbert/Ramme, Manfred. 1988, $13 \mathrm{ff}$.

Maiers, Wolfgang. 1999. Funktional-historische Analyse. In Historisch-kritisches Wörterbuch des Marxismus Band 4, herausgegeben von Wolfgang Fritz Haug. Berlin/Hamburg: Argument Verlag.

Maiers, Wolfgang. 2006. Präsentative Symbolik in der menschlichen Stammesgeschichte. Vorbemerkung zu Ekkehard Jürgens. Forum Kritische Psychologie 49, $58 \mathrm{ff}$.

Maiers, Wolfgang. 2019. Menschliche Subjektivität und Natur. Wissenschaftlicher Humanismus bei Karl Marx und in der Kritischen Psychologie. In Marx bleibt aktuell. Perspektiven für die Einzelwissenschaften, herausgegeben von Wolfgang Maiers und Renatus Schenkel, 131-159. Halle (Saale): Mitteldeutscher Verlag.

Maiers, Wolfgang, und Morus Markard. 1978. Zur Existenzweise marxistischer Wissenschaft unter dem Berufsverbot. Forum Kritische Psychologie 3, 7-12.

Maletzke, Gerhard. 1963. Psychologie der Massenkommunikation. Theorie und Systematik. Hamburg: Verlag Hans Bredow-Institut.

McLuhan, Marshall, und Bruce R. Powers. 1995. The Global Village: Transformations in World Life and Media in the 21st Century. Oxford University Press.

Medienanstalten, Die. 2019. Staatsvertrag für Rundfunk und Telemedien in der Fassung vom 01. Mai 2019, auf: www.die-medienanstalten.de

Meyen, Michael. 2017. Die (doppelt) konservative Wende in der Kommunikationswissenschaft. Vortrag auf der Gründungstagung des Netzwerks Kritische Kommunikationswissenschaft, Ludwig-Maximilians-Universität München 30.11.-1.12.2017, online: https:// www.youtube.com/watch?v=Qtium9RjnpQ\&feature=youtu.be ( $\mathrm{min}$. 0:53:04 bis 1:15:30). Zugegriffen: 15. November 2019.

Merten, Klaus. 1982. Wirkungen der Massenkommunikation. Ein theoretisch-methodischer Problemaufriß. Publizistik 27 (1-2).

Prigogine, Ilya. 1985. Vom Sein zum Werden. Zeit und Komplexität in den Naturwissenschaften. München/Zürich: Piper.

Sevignani, Sebastian. 2018. „Historisch-Materialistische Medien- und Kommunikationstheorie 2.0". Maske und Kothurn: Internationale Beiträge zur Theater-, Film- und Medienwissenschaft 64 (1/2): 59-88.

Schenkel, Renatus. 1988. Kommunikation und Wirkung. Gesellschaftliche und psychische Voraussetzungen medialer Kommunikation. Frankfurt am Main/New York: Campus. 
Schenkel, Renatus. 2015. Fankultur im medialen Zeitalter. In Fußball global. Ein Spiel dauert länger als 90 Minuten. Interdisziplinäre Beiträge zu Phänomenen des Fußballsports, herausgegeben von Peter Ulrich Wendt, Stefan Roggenthin, Renatus Schenkel, Titus Simon und Michael Thomas, 101ff. Halle (Saale): Mitteldeutscher Verlag.

Schenkel, Renatus. 2018. Vorwort. in: Engelmann, Nikolay. 2018. Virtual Reality Gaming Potential der Technologie für die Welt der digitalen Spiele, $6 \mathrm{ff}$.

Schenkel, Renatus. 2019. Marx und Medien. ,Medialisierung' als zentraler Trend im Spätkapitalismus? In Marx bleibt aktuell. Perspektiven für die Einzelwissenschaften, herausgegeben von Wolfgang Maiers und Renatus Schenkel, Halle (Saale): Mitteldeutscher Verlag.

Schurig, Volker. 1975. Naturgeschichte des Psychischen 1 und 2.

Sevignani, Sebastian. 2018. Historisch-Materialistische Medien- und Kommunikationstheorie, in: Schröder/Heilmann, $9 \mathrm{ff}$.

Schröder, Jens, Heilmann, Till A. 2018. Maske und Koturn. Internationale Beiträge zur Theater-, Film- und Medienwissenschaft 64 (1-2)

Shannon, Claude E., Weaver, Warren. 1963. Mathematical Theory of Communications. University of Illinois Press.

Struve, Ulrich. 1999. Anmerkungen zur Kaspar-Hauser-Rezeption: Ein bibliographischer Bericht (1840-1998), https:// www.mtholyoke.edu/courses/gdavis/325kaspar/kasparbib.htm

Württembergische Landesbibliothek Stuttgart. 2019. Neues Testament, https://www.wlbstuttgart.de/referate/theologie/swvotxt/kwbint.pdf, Abruf 05.03.2019

ZDF. 2018. ZDF-Staatsvertrag vom 31. August 1991, in der Fassung des Einundzwanzigsten Staatsvertrages zur Änderung rundfunkrechtlicher Staatsverträge (Einundzwanzigster Rundfunkänderungsstaatsvertrag) in Kraft seit 25. Mai 2018.

\section{Open Access}

Dieser Beitrag erscheint unter der Creative-Commons-Lizenz CC-BY-SA 4.0:

https://creativecommons.org/licenses/by-sa/4.0/ 